\title{
Élevage bovin en plantation industrielle de palmiers à huile au Cameroun. I. Étude de la flore P. Gaullier 1 et mesure des biomasses
}

\begin{abstract}
GAULLIER (P.). Élevage bovin en plantation industrielle de palmiers à huile au Cameroun. I. Etude de la flore et mesure des biomasses. Revue Elev. Méd. vét. Pays trop., 1990, 43 (3) : 401-408

Un élevage bovin de race trypanotolérante (N'Dama) a été créé à partir de 1979 dans une palmeraie industrielle de la province du littoral au Cameroun. Une étude des pâturages a été entreprise, dont la première phase était une enquête agrostologique dans les différentes tranches d'âge de la palmeraie. Elle a permis de faire une évaluation globale de la productivité fourragère par mesure des biomasses totales et utiles. L'influence des travaux d'entretien de la palmeraie ainsi que les caractéristiques originales de ces pâturages sont examinées ; l'étude de leur dynamique et l'analyse de leur évolution sous exploitation sont amorcées. Mots clés : Élevage bovin - Palmeraie industrielle - Pâturages - Productivité des terres - Biomasse - Cameroun.
\end{abstract}

\section{INTRODUCTION}

Avec le démarrage, après 1960 , de vastes programmes de plantations industrielles de palmiers à huile (Elaeis guineensis Jacq.) dans les pays côtiers d'Afrique, est apparue l'idée de développer dans ces unités une activité annexe d'élevage à l'exemple de celle pratiquée depuis longtemps sous les cocoteraies du Pacifique (Philippines, Nouvelles-Hébrides...). Dans les deux cas, il s'agit d'utiliser rationnellement le couvert herbacé qui se développe sous les palmiers, mais la nature de ce couvert varie considérablement, en fonction notamment de l'ombrage. Dans les cocoteraies, l'éclairement au sol est généralement supérieur à 50 p. 100 et permet au pâturage sous-jacent de supporter des charges de 1 à 2 UBT (unité de bétail tropical) à l'hectare ou de cultiver des fourrages capables de nourrir deux fois plus d'animaux (5). Dans les plantations de palmiers à huile adultes, l'ombrage est presque total et le pâturage d'ombre subsistant ne peut nourrir, au mieux, que 1 UBT à l'hectare (6). Aucune culture fourragère n'y est possible et la valeur du pâturage est essentiellement tributaire de sa composition floristique, laquelle évolue au cours des vingt-cinq années de vie utile de la palmeraie. La localisation dans les régions équatoriales chaudes et pluvieuses limite le choix du bétail aux races trypanotolérantes.

1. Agence Nationale de Développement de l'Élevage, BP 1509, Bangui, République Centrafricaine.

Reçu le 23.1.1990, accepté le 27.4.1990.

\section{MATÉRIEL ET MÉTHODE}

Les résultats dont il est fait état ici ont été obtenus avec l'élevage expérimental de la Socaipalm à la plantation de M'Bongo au Cameroun, de 1979 à 1985.

\section{Cadre de l'étude}

La plantation de M'Bongo est située à $30 \mathrm{~km}$ au sudest de Douala, sur l'ancienne; nationale 1 DoualaEdéa, après le bac de la Dibamba. Elle est établie sur des sols ferralitiques développés sur des sédiments du pleistocène, de texture argilo-sableuse, acides et pauvres chimiquement. Le planting a été fait après défrichement, brûlage et andainage de la forêt dense, et, d'une manière générale, les quantités de bois résiduel sont encore importantes. Le climat est de type équatorial à forte pluviosité groupée en une seule saison de huit à neuf mois; la saison sèche ne dépasse pas, normalement, six semaines sans précipitations et l'hygrométrie, toujours supérieure à 60 p. 100, fait qu'il existe tout au long de l'année, sous la palmeraie, des pluies occultes par condensation sur les palmes (tabl. I).

Dans les blocs âgés de 4 à 10 ans en 1979, la couverture initiale de Pueraria*' semée au planting était en grande partie remplacée par des graminées sciaphiles, et de nombreuses espèces d'origine forestière existaient sur les andains.

\section{Étude de la composition botanique}

Dès le début du projet, en juillet 1979, une enquête botanique a été réalisée systématiquement avec constitution d'un herbier, limité dans un premier temps aux plantes présentant a priori un intérêt pour l'élevage (Poaceae), puis à toutes celles auxquelles le bétail s'est intéressé. Les plantes, au nombre de cinquante, ont été déterminées par J.P. LEBRUN de l'IEMVT, à l'exception de six d'entre elles: il s'agit des herbacées portant les numéros d'herbier 25 et 27 , et les ligneuses numérotées $32,33,37$ et 46 qui 'n'ont pu être déterminées, fleurs ou fruits n'ayant pas été trouvés. Seules

\footnotetext{
* Pueraria phaseoloides (Roxb.) Benth., var. javanica (Benth.) Bak., introduite [syn. : $P$. javanica (Benth.) Benth.]
} 


\section{P. Gaullier}

TABLEAU I Climatologie de la région de M'Bongo.

\begin{tabular}{|c|c|c|c|c|c|c|c|c|c|c|c|c|c|}
\hline Données climatiques & J & $\mathrm{F}$ & M & A & M & J & $J$ & A & $S$ & 0 & $N$ & $\mathrm{D}$ & Total \\
\hline Pluviométrie (mm) & 20,2 & 83,6 & 185,4 & 277,0 & 289,8 & 444,7 & 555,1 & 660,5 & 570,9 & 442,5 & 183,0 & 27,1 & 3750,2 \\
\hline Ensoleillement (heures) & 206,8 & 196,7 & 180,9 & 184,6 & 182,1 & 118,0 & 68,8 & 50,6 & 79,0 & 127,9 & 173,7 & 203,5 & 1772,6 \\
\hline Température moyenne $\left({ }^{\circ} \mathrm{C}\right)$ & 26,9 & 27,4 & 27,2 & 27,1 & 26,9 & 25,7 & 24,5 & 24,3 & 25,0 & 25,7 & 26,5 & 26,7 & 26,2 \\
\hline
\end{tabular}

trois d'entre elles participent significativement à la constitution du bol alimentaire; elles seront signalées par leurs numéros dans l'étude des valeurs bromatologiques.

La publication conjointe IRHO-Shell-Cameroun (7) sur la flore adventice de la station voisine de la Dibamba a servi de référence pour le repérage des principales espèces non pastorales de ce domaine botanique extrêmement riche et diversifié. En effet, TCHOUME, dans son étude sur les palmeraies de Côte-d'lvoire**, citée par ROMBAULT (10), dénombre 325 espèces différentes, et la flore des palmeraies camerounaises est certainement aussi riche; cette étude sera donc loin d'être exhaustive, encore que les plantes citées sont généralement celles qui fournissent la quasitotalité de la biomasse sous palmiers.

\section{Mesure des biomasses}

Sur le terrain, deux séries d'échantillonnage ont été menées selon la méthode des placeaux situés par tirage au sort dans les parcelles des palmeraies (de 25 à 100 ha), elles-mêmes choisies au hasard, à raison de deux parcelles par tranche d'âge de palmeraie (chaque tranche regroupant deux années de plantation). Les récoltes des placeaux n'étaient jamais faites avant une période de repousse inférieure à deux mois après exploitation ou gyrobroyage (tabl. II). L'échantillonnage d'une parcelle est réalisé par la pose aléatoire de cinq placeaux de $4 \mathrm{~m}^{2}(2 \mathrm{~m} \times 2 \mathrm{~m})$, récoltés au cours d'une même matinée.

La délimitation du placeau est faite à l'aide d'une cordelette graduée, à partir du palmier-repère tiré au sort (tirage non exhaustif dans chacune des 5 lignes de 29 palmiers sélectionnées par tirage exhaustif), sur l'un des 16 emplacements possibles, autour de chaque palmier lui-même choisi au hasard (huit emplacements côté interligne et huit autres côté andain pour chaque palmier).

La récolte est faite avec une paire de cisailles de jardinier et toutes les plantes sont coupées au ras du collet, sauf pour les ligneux dont seules les parties herbacées sont récoltées.

** TCHOUME (M.). Étude de la végétation adventice des palmeraies à Elaeis guineensis Jacq, en Côte-d'lvoire forestière. Université d'Abidjan, Faculté des Sciences.
TABLEAU II Échantillonnage pour l'étude des biomasses à $M$ 'Bongo.

\begin{tabular}{|c|c|c|c|c|c|}
\hline \multirow{2}{*}{$\begin{array}{l}\text { Date de } \\
\text { récolte des } \\
5 \text { placeaux }\end{array}$} & \multicolumn{2}{|c|}{ Numéro } & \multirow{2}{*}{$-\begin{array}{c}\text { Année } \\
\text { du } \\
\text { "planting" }\end{array}$} & \multicolumn{2}{|c|}{$\begin{array}{l}\text { Dernières interventions } \\
\text { (parcelles échantillonnées) }\end{array}$} \\
\hline & Bloc & Parcelle & & Date & Nature \\
\hline 19.11 .1983 & 04 & 5 & 1970 & 5.1983 & Pâturage \\
\hline 18.11 .1983 & 40 & 4 & 1969 & - & $\begin{array}{l}\text { Non exploité depuis } \\
\text { février } 1982\end{array}$ \\
\hline 23.11 .1983 & 64 & 6 & 1972 & 7.1983 & Pâturage \\
\hline 28.11 .1983 & 80 & 2 & 1973 & 7.1983 & Pâturage \\
\hline 24.11 .1983 & 63 & 4 & 1973 & - & $\begin{array}{l}\text { Entretien antérieur à } \\
\text { juillet } 1983\end{array}$ \\
\hline 25.11 .1983 & 67 & 6 & 1975 & 9.1983 & Pâturage \\
\hline 23.5 .1984 & 04 & 5 & 1970 & 12.1983 & $\begin{array}{l}\text { Pâturage et gyro- } \\
\text { broyage }\end{array}$ \\
\hline 5.7 .1984 & 40 & 4 & 1969 & 3.1984 & Pâturage \\
\hline 24.5 .1984 & 64 & 6 & 1972 & 2.1984 & Pâturage \\
\hline 21.4 .1984 & 54 & 2 & 1971 & 11.1983 & $\begin{array}{l}\text { Pâturage et gyro- } \\
\text { broyage }\end{array}$ \\
\hline 7.6.1984 & 80 & 2 & 1973 & 3.1984 & $\begin{array}{l}\text { Pâturage et gyro- } \\
\text { broyage }\end{array}$ \\
\hline 5.6 .1984 & 63 & 4 & 1973 & 2.1984 & Gyrobroyage \\
\hline 25.4 .1984 & D4 & 4 & 1975 & 12.1983 & $\begin{array}{l}\text { Gyrobroyage (jamais } \\
\text { pâturé) }\end{array}$ \\
\hline 25.5 .1984 & 67 & 6 & 1975 & 3.1984 & $\begin{array}{l}\text { Pâturage et gyro- } \\
\text { broyage }\end{array}$ \\
\hline 26.4 .1981 & c5 & 3 & 1977 & 12.1983 & $\begin{array}{l}\text { Entretien manuel } \\
\text { (jamais pâturé) }\end{array}$ \\
\hline
\end{tabular}

On procède ensuite à la pesée globale de la récolte des cinq placeaux de la parcelle, puis au tri des plantes consommables, et enfin à la pesée en vert des récoltes de chacune de ces plantes. Ces échantillons sont ensuite séchés 24 heures en étuve de 1800 watts, puis pesés.

Dans la première série d'échantillonnage réalisée en novembre 1983, seules les pesées en vert ont été exécutées; dans la seconde série réalisée d'avril à juin 1984, les échantillons ont été pesés en vert et en sec sur une balance électronique (précise à $0,5 \mathrm{~g}$ ) à la station IRA de Dibamba. Dans les deux cas, on a donc déduit, pour chaque parcelle, les parties non consommables de la biomasse totale et obtenu ainsi la biomasse utile. Les taux de matière sèche de la seconde série ont été extrapolés à la première car on ne disposait pas en 1983 d'un matériel suffisamment précis. 


\section{RESULTATS}

\section{Description de la flore}

L'établissement des blocs de palmeraies industrielles sur des défrichements récents de forêt dense entraîne un changement brutal de la flore locale. D'après les résultats des études menées par KHAN (8) en forêt dense du Sud-Ouest ivoirien, la classification suivante, en trois types de végétation, est proposée :

A. Les plantes issues de formations exogènes, c'est-àdire non forestières, de type savanicole, qui profitent plus ou moins rapidement, selon le degré d'isolement du bloc, des nouvelles conditions d'éclairement au sol.

B. Les espèces colonisantes issues des bordures ou clairières forestières où elles forment les premières phases de reconstitution de la forêt dégradée.

C. Les espèces résiduelles de la forêt dense, qui n'ont jamais complètement disparu et qui retrouvent peu à peu des conditions favorables à leur développement lorsque le couvert des palmiers s'installe.

On observe que ces trois groupes de plantes tendent à s'installer chronologiquement dans cet ordre au cours des quinze premières années de croissance de la strate arborée constituée par les palmiers, et qu'elles se développent au moins deux à deux, concurrentiellement, durant cette période.

\section{A. Les plantes issues de formations exogènes}

Ce groupe est presque uniquement constitué de plantes herbacées, pour la plupart bien consommées par le bétail ; on y trouve bon nombre de graminées classiques des pâturages soudano-guinéens, et aussi des monocotylédones typiques des régions chaudes et humides (au moins saisonnièrement) telles que les commélinacées, et diverses petites dicotylédones annuelles dont l'apport fourrager est marginal; seule légumineuse de ce groupe, le Pueraria de couverture n'est plus la ressource fourragère de base. La plupart des fougères rencontrées sous palmeraie sont classées ici, de même que les trois espèces suffrutescentes et envahissantes.

\section{a. Les graminées (Poaceae)}

Acroceras amplectens Stapf

Andropogon gayanus Kunth

Brachiaria mutica (Forsk.) Stapf
Digitaria horizontalis Willd.

Eleusine indica (L.) Gaertn.

Panicum brevifolium L.

Panicum maximum Jacq.

Paspalum paniculatum L.

Paspalum scrobiculatum L.

Pennisetum polystachyon (L.) Schult.

Pennisetum purpureum Schum.

Perotis indica (L.) O. Ktze.

Setaria megaphylla (Steud.) Dur. et Schinz

Sporobolus pyramidalis $\mathrm{P}$. Beauv.

Schizachyrium sp.

Elles sont toutes héliophiles, donc essentiellement localisées sur les allées de récolte et bordures de parcelles, qui représentent environ 5 p. 100 des surfaces. Leur rythme végétatif y est deux à trois fois plus rapide que celui de la végétation d'ombre sous palmiers.

\section{b. Les autres monocotylédones}

Famille des Commelinaceae

Aneilema beniniense (P. Beauv.) Kunth

Commelina africana $\mathrm{L}$.

Commelina diffusa Burm. f.

Commelina benghalensis $\mathrm{L}$.

\section{Famille des Cyperaceae}

Cyperus tenuiculmis Boeck. var. schweinfurthianus (Boeck.) Hooper

Cyperus fertilis Boeck.

Contrairement aux cypéracées, faiblement consommées et de peu de valeur, les Commélinacées sont des plantes fourragères intéressantes.

\section{c. Les herbacées diverses}

\section{Acanthaceae}

Asystasia gangetica (L.) T. Anders.

Brillantaisia lamium (Nees) Benth. 


\section{Amaranthaceae}

Cyathula achyranthoides (H.B. et K.) Moq.

Achyranthes aspera L.

\section{Asteraceae}

Emilia javanica (Burm. f.) C.B. Robins.

Elephantopus mollis Kunth

\section{Fabaceae}

Pueraria phaseoloides (Roxb.) Benth. var. javanica (Benth.) Bak. (introduit)

\section{Melastomataceae}

Dissotis prostrata (Thonn.) Hook. f. et quelques autres espèces du même genre

\section{Rubiaceae}

Sabicea calycina Benth.

Oldenlandia lancifolia (Schum.) DC.

En dehors de Pueraria, ces plantes fournissent une végétation pionnière sur sols lessivés et déstructurés (Oldenlandia, Dissotis) et certaines sont indésirables dans les pâturages classiques (Elephantopus).

\section{d. Les fougères (ptéridophytes)}

Nephrolepis undulata (Afz. ex Sw.) J. Sm. (Davalliaceae)

Pityrogramma calomelanos (L.) Link (Adiantaceae, introduite)

Pteridium aquilinum (L.) Kuhn (Dennstaedtiaceae)

Gleichenia linearis (Burm.) C.B. Clarke (Gleicheniaceae)

Selaginella myosurus (Sw.) Alston (Selaginellaceae)

Elles sont sans intérêt pour l'élevage. Leur comportement héliophile ne semble pas concurrencer les espèces utiles sous palmiers, comme elles peuvent le faire dans des pâtures ouvertes.

\section{e. Les espèces suffrutescentes***}

Chromolaena odorata (L.) R.M. King et Robinson [bas. : Eupatorium odoratum L. (Asteraceae)]

Lantana camara L. (Verbenaceae)

Mimosa pudica L. (Mimosaceae)

Considérées comme de véritables "pestes " des palmeraies, ces plantes sont aussi dangereuses du point de vue pastoral que du point de vue agronomique.

\section{B. Les espèces colonisantes d'origine périforestière}

Dans ce groupe, ce sont les espèces ligneuses, arbres et arbustes héliophiles à croissance et déploiement (élargissement des cimes) rapides, qui dominent. Chronologiquement, ils sont aussi les premiers agents de salissement de la plantation, la plante de couverture retardant l'installation des herbacées pionnières jusqu'à extension de l'ombrage. Dans l'intervalle apparaissent des espèces lianescentes et suffrutescentes qui sont à rattacher à ce groupe.

\section{a. Les espèces ligneuses}

Musanga cercropioides $\mathrm{R}$. Br. ou parasolier (Moraceae)

Alchornea cordifolia (Schum. et Thonn.) Müll. Arg. (Euphorbiaceae)

Anthocleista sp. (Loganiaceae)

Clappertonia ficifolia (Willd.) Decne. (Tiliaceae)

Macaranga hurifolia Beille (Euphorbiaceae)

Tetracera alnifolia Willd. (Dilleniaceae)

Tetrorchidium didymostemon (Baill.) Pax et Hoffm. (Euphorbiaceae)

Vernonia conferta Benth. (Asteraceae)

Phyllanthus muellerianus (Euphorbiaceae)

Les feuilles de ces arbustes sont toutes plus ou moins consommées par le bétail et il est remarquable de ne trouver en palmeraie aucune plante délaissée de ce type, telle Harungana madagascariensis Lam., pourtant abondante sur défrichements anciens dans la région.

*** Plantes à tiges plus ou moins lignifiées et de petite taille. 


\section{b. Les espèces lianescentes}

Cogniauxia podolaena Baill. (Cucurbitaceae)

Mikania cordata (Burm. f.) B.L. Robins. (Asteraceae)

Stephania sp. (Menispermaceae)

Elles sont dangereuses pour les palmiers, même adultes, car elles peuvent étouffer la cime. Sur les andains, Cogniauxia et Stephania sp. sont faiblement consommées.

\section{c. Les espèces suffrutescentes}

Ancistrocarpus densispinosus Oliv. (Tiliaceae)

Clerodendrum splendens G. Don (Verbenaceae)

Haumania danckelmaniana (J. Br. et K. Schum.) MilneRedh. (Maranthaceae)

Parmi ces plantes, peu appétées par le bétail, seule Haumania, rhizomateuse et vivace, pose des problèmes de contrôle car sa destruction chimique n'a pas encore été mise au point.

\section{d. Les espèces herbacées}

Ce sont des graminées sciaphiles**** qui remplacent Pueraria sous l'ombrage des palmiers:

Megastachya mucronata (Poir.) P. Beauv.

Axonopus compressus (Sw.) P. Beauv.

Paspalum conjugatum Berg.

Panicum laxum Sw. (introduite)

et une fougère à comportement sciaphile, Nephrolepis biserrata Schott (Davalliaceae), qui, contrairement à celles du groupe $A$, peut réduire par son extension la biomasse utile disponible pour l'élevage sous palmiers. On classera également ici une petite légumineuse d'ombre, Desmodium adscendens Sw. (Fabaceae), bien appétée.

\section{Les espèces résiduelles de forêt dense}

Ces plantes représentent les traces d'un ensemble botanique très riche et mal connu. C'est le domaine des grandes essences forestières utiles à croissance

**** Se dit des plantes qui, se développent de préférence à l'ombre. lente qui ont pratiquement disparu du milieu domestique, mais aussi d'espèces strictement sciaphiles, de modeste développement et relativement abondantes, qui peuvent être classées en subligneuses et suffrutescentes.

\section{a. Les espèces suffrutescentes}

Aframomum sceptrum (Oliv. et Hanb.) K. Schum. (Zingiberaceae)

Aframomum subsericeum (Oliv. et Hanb.) K. Schum. (Zingiberaceae)

Costus afer Ker-Gawl. (Zingiberaceae)

Palisota hirsuta (Thunb.) K. Schum. (Commelinaceae)

Elles représentent la plus grande part de la biomasse fournie par ce groupe et sont relativement envahissantes car rhizomateuses: Costus et Pasilota sont prélevées au niveau des feuilles, mais peuvent être délaissées quand elles forment l'essentiel de la végétation des andains comme c'est souvent le cas.

\section{b. Les espèces subligneuses}

Mussaenda landophioides Wernham (Rubiaceae)

Sabicea sp. (Rubiaceae)

Urera cordifolia Engler (Urticaceae)

Elles sont toutes plus ou moins lianescentes et leurs feuilles sont consommables.

\section{c. Les espèces forestières}

Lophira alata Banks ex Gaertn. f. (Ochnaceae), communément azobé, est l'exemple type de cette végétation totalement étrangère au domaine pastoral. Des semis d'azobé peuvent être abondants en lisière forestière.

\section{Les biomasses}

\section{Composition}

La part de la biomasse utile (consommée par le bétail) dans la biomasse totale (poids de matière verte) varie approximativement de un à deux tiers. On peut faire les remarques suivantes (tabl. III) :

- cette proportion ne dépend pas de l'âge de la palmeraie ; 


\section{P. Gaullier}

TABLEAU III Part de la biomasse utile dans la biomasse totale.

\begin{tabular}{|c|c|c|c|c|c|c|}
\hline \multicolumn{3}{|c|}{ Palmeraie } & \multicolumn{4}{c|}{ Pâturage sous palmeraie } \\
\hline $\begin{array}{c}N^{\circ} \\
\text { bloc }\end{array}$ & $\begin{array}{c}N^{\circ} \\
\text { parcelle }\end{array}$ & $\begin{array}{c}\text { Age au } \\
\text { prélèvement } \\
\text { (ans) }\end{array}$ & $\begin{array}{c}\text { Age du recrú } \\
\text { végétal } \\
\text { (mois) }\end{array}$ & $\begin{array}{c}\text { Biom. totale } \\
\text { (kg/ha) }\end{array}$ & $\begin{array}{c}\text { Biom. utile } \\
\text { (kg/ha) }\end{array}$ & $\begin{array}{c}\text { BU/BT } \\
\text { (p. 100) }\end{array}$ \\
\hline 40 & 4 & 15 & 4 & 1584 & 453,5 & 28,6 \\
40 & 4 & 14 & $?$ & 1625 & 1000 & 61,5 \\
04 & 5 & 14 & 6 & 2629,5 & 1651 & 62,8 \\
04 & 5 & 13 & 5 & 3500 & 2425 & 69,3 \\
54 & 2 & 12 & 5 & 2923 & 1689 & 57,8 \\
64 & 6 & 12 & 3,5 & 2565,5 & 1179,5 & 45,9 \\
64 & 6 & 11 & 4 & 3000 & 1150 & 38,3 \\
80 & 2 & 11 & 3 & 3482,5 & 1725 & 49,5 \\
63 & 4 & 11 & 4 & 2220,5 & 1497,5 & 67,4 \\
80 & 2 & 10 & 4 & 4100 & 3200 & 78,0 \\
63 & 4 & 10 & $?$ & 2900 & 2000 & 68,9 \\
D4 & 4 & 9 & 4 & 10003,5 & 1298 & 12,9 \\
67 & 6 & 9 & 2 & 3004,5 & 938 & 31,2 \\
67 & 6 & 8 & 2 & 1900 & 600 & 31,5 \\
\hline \multicolumn{7}{|l|}{ Moyenne } \\
\hline
\end{tabular}

- il n'y a pas de corrélation apparente entre les quantités respectives de biomasse et l'âge des recrûs végétaux sous palmiers ;

- un faible pourcentage de biomasse utile n'implique pas toujours un faible disponible (cas du bloc D4, parcelle 4).

Le sous-étage végétal de la palmeraie industrielle est un milieu artificialisé où l'intervention de l'homme perturbe les processus de régénération spontanée. Or, ces interventions visent à optimiser le rendement des palmiers et non celui du pâturage. Le facteur premier de variation quantitative et qualitative de la biomasse est le degré d'ombrage, et celui-ci varie fortement sous palmeraie adulte avec la fréquence des élagages. D'autre part, les entretiens par rabattage manuel et gyrobroyage des interlignes tendent à faire disparaître certaines espèces, et d'abord les ligneux. Ces interventions ont donc une influence déterminante, difficilement mesurable, sur la valeur fourragère de la biomasse.

Une autre caractéristique de ce pâturage d'ombre est la lenteur de la repousse des herbacées, qui y atteignent un développement optimal en six à huit semaines et demeurent à un stade jeune prolongé, sans floraison pour la plupart, jusqu'au-delà de trois mois. La biomasse varie donc peu quantitativement, et même qualitativement, entre deux et quatre mois d'âge après exploitation.

Enfin, certaines plantes adventices, non appétées par le bétail et à fort pouvoir colonisant (Chromolaena,
Lantana, Haumania), peuvent gonfler le volume total de la biomasse sans pour autant éliminer, sinon partiellement, la flore fourragère.

Le tableau IV récapitule les quantités récoltées par parcelle de chacune des plantes participant à la constitution de la biomasse utile, biomasse fourragère. Elles sont réparties dans chacune des classes botaniques définies par l'étude de la flore afin d'amorcer l'analyse de l'évolution du pâturage dans le temps.

\section{Évolution}

A partir du tableau $V$, illustré par la figure 1 , on constate que l'hypothèse d'une dynamique liée aux écotypes se vérifie: le groupe des plantes d'origine forestière $(C)$ prend une importance croissante de la $9 e$ à la $14 \mathrm{e}$ année. Sous l'ombrage de plus en plus dense des couronnes jointives des palmiers, ces plantes retrouvent des conditions favorables à leur développement. Cet accroissement se fait naturellement au détriment des plantes du groupe $A$ de comportement héliophile tandis que le groupe $B$ des plantes de recolonisation forestière tend à rester seul en compétition. A noter que les ligneux des trois groupes tendent à disparaître du fait de l'entretien.

On observe cependant une brusque inversion de cette évolution en $15 \mathrm{e}$ année, qu'il faut interpréter prudemment compte tenu de la faible taille de l'échantillon (une seule série de prélèvements, soit 5 placeaux) : elle est a priori normale car, l'homogénéité génétique des palmiers n'étant pas parfaite, ils se différencient par la taille en fin de carrière, et peu à peu la discontinuité de la strate végétale haute (couronnes des palmiers) s'installe, favorisant une recrudescence de l'éclairement au sol. Le mouvement devrait s'opérer beaucoup plus rapidement entre 15 et 20 ans d'âge de la palmeraie.

\section{DISCUSSION ET CONCLUSION}

La méthode adoptée pour l'étude du pâturage sous palmeraie à M'Bongo privilégie les mesures de biomasse utile au détriment de l'analyse fréquentielle de la flore. La raison de ce choix est le manque de moyens et de personnel pour la mise en oeuvre d'un dispositif lourd, qui reste d'ailleurs à définir pour être en mesure d'appréhender l'hétérogénéité et la variabilité de ces formations végétales. En effet, 70 prélèvements, totalisant $350 \mathrm{~m}^{2}$ de surface récoltée, n'ont permis d'intercepter que 32 des 50 plantes recensées et analysées, soit $64 \mathrm{p} .100$ seulement du cortège floristique pastoral, lui-même arbitrairement limité à 50 plantes. Certes, beaucoup de graminées héliophiles 
TABLEAU IV Composition de la biomasse utile préséchée. Répartition par types de végétation selon l'âge des palmeraies et calcul des matières sèches.

\begin{tabular}{|c|c|c|c|c|c|c|c|c|c|c|c|c|c|c|c|c|c|c|c|}
\hline \multirow{3}{*}{$\begin{array}{l}\text { Classe } \\
\text { bota- } \\
\text { nique }\end{array}$} & \multirow{3}{*}{$\begin{array}{c}N^{\circ} \\
\text { herbier }\end{array}$} & \multirow{3}{*}{ Plante } & \multicolumn{17}{|c|}{ Age des palmiers et numéro des blocs et parcelles } \\
\hline & & & \multirow{2}{*}{$\frac{15 \text { ans }}{40-4}$} & \multicolumn{2}{|c|}{14 ans } & \multirow{2}{*}{\begin{tabular}{|c|}
13 ans \\
$04-5$ \\
\end{tabular}} & \multicolumn{2}{|c|}{12 ans } & \multicolumn{3}{|c|}{11 ans } & \multicolumn{2}{|c|}{10 ans } & \multicolumn{2}{|c|}{9 ans } & \multirow{2}{*}{\begin{tabular}{|c|}
8 ans \\
$67-6$
\end{tabular}} & \multirow{2}{*}{\begin{tabular}{|c|} 
Total pré- \\
séchés \\
$(\mathrm{g})$
\end{tabular}} & \multirow{2}{*}{$\begin{array}{c}\text { Total } \\
\text { MS } \\
(\mathrm{g})\end{array}$} & \multirow{2}{*}{$\begin{array}{c}\text { Mat. } \\
\text { sèches } \\
\text { (kg/ha) }\end{array}$} \\
\hline & & & & $40-4$ & $04-5$ & & $64-6$ & $54-2$ & $64-6$ & $80-2$ & $63-4$ & $80-2$ & $63-4$ & $04-4$ & $67-6$ & & & & \\
\hline \multirow{11}{*}{$\begin{array}{c}\text { Aa } \\
\text { b } \\
\text { c }\end{array}$} & 11 & Panicum brevifolium & - & 40,5 & - & - & - & - & 23,0 & 24,5 & - & 38,5 & 40,0 & - & - & 12,0 & 178.5 & 164.0 & 585 \\
\hline & 5 & Pennisetum polytachion & - & - & - & 63,5 & - & - & - & - & - & - & - & 201,5 & - & - & 265,0 & 246,0 & 8,78 \\
\hline & 23 & Anellema beniniense & 16,0 & 11,5 & 19,0 & 72,0 & - & - & - & 56,0 & - & 87,5 & 137,0 & - & - & - & 399,0 & 359,5 & 12,84 \\
\hline & 22 & Cyperus tenuiculmis & - & 12,0 & 9,0 & - & 14,5 & - & - & - & - & - & - & 11,0 & 10,5 & - & 57,0 & 52,5 & 1,87 \\
\hline & 43 & Asystasia gangetica & - & $\bar{n}$ & 1,0 & - & - & 26,5 & - & - & - & - & - & - & - & - & 27,5 & 25,0 & 0,89 \\
\hline & $\begin{array}{l}26 \\
18\end{array}$ & $\begin{array}{l}\text { Cyathula achyranthoides } \\
\text { Emilia javanica }\end{array}$ & - & 20,0 & $\overline{14} 0$ & 48,5 & $\overline{590}$ & $\overline{2}$ & $\overline{10}$ & 5,0 & - & 38,5 & - & - & - & 12,0 & 124,0 & 112,5 & 4,02 \\
\hline & 14 & Pueraria phaseoloides & - & - & 14,0 & - & 0,0 & , & 18,5 & - & - & 4,0 & - & - & - & - & 98,0 & 0 & 3,18 \\
\hline & & var. Javanica & 111,0 & - & 17,0 & 10,0 & 61,5 & 13,5 & - & 316,0 & 13,5 & 103,5 & 8,0 & 145,0 & - & 12,0 & 811,0 & 743,5 & 26,55 \\
\hline & 20 & Dissotis prostrata & - & - & - & - & - & 21,5 & - & - & - & - & 110,5 & - & - & - & 132,0 & 5 & 4,27 \\
\hline & 21 & Sabicea calycina & 6,0 & 65,0 & 33,5 & - & 51,0 & 85,5 & - & - & 70,5 & 6,0 & 207,5 & - & - & - & 525,0 & 48 & 17,34 \\
\hline & $\begin{array}{l}19 \\
27\end{array}$ & $\begin{array}{l}\text { Oldenlandia lancifolia } \\
\text { Rublacée indéterminée }\end{array}$ & $\overline{7} 5$ & 2,0 & $\overline{38} 0$ & - & $\overline{14} 5$ & - & - & - & $\overline{17}$ & 87,0 & 4,5 & - & - & - & 93,5 & 5 & 3,05 \\
\hline \multirow[t]{8}{*}{$\mathrm{Ba}$} & 34 & Alchornea cordifolia & - & - & Do,0 & - & 14,5 & $\overrightarrow{21,5}$ & - & $\overline{9}, 0$ & 11,5 & $23 \overline{7}, 5$ & - & $\begin{array}{l}42,5 \\
26,0\end{array}$ & $\begin{array}{l}44,0 \\
98,5\end{array}$ & $\overline{19}$ & $\begin{array}{l}16 \\
41\end{array}$ & & 5 \\
\hline & 31 & Anthocleista sp. & - & 14,0 & - & 50,0 & - & 47,0 & - & - & - & $-\infty$ & - & - & - & - & 111,0 & 101,0 & $\begin{array}{l}13,30 \\
3,60\end{array}$ \\
\hline & 29 & Macaranga hurifolia & - & - & - & 60.5 & 85,0 & - & 28,5 & - & - & - & - & 2,5 & - & - & 176,5 & 15 & 5,66 \\
\hline & $\begin{array}{l}38 \\
40\end{array}$ & $\begin{array}{l}\text { Tetracera alnifolia } \\
\text { Tetrochidium }\end{array}$ & - & - & - & - & 115,0 & - & 107,0 & - & - & - & - & - & - & - & 222,0 & 20 & 7,18 \\
\hline & & didymostemon & - & 15,0 & - & - & - & - & - & - & - & - & - & - & - & - & 15,0 & 13 & 0,48 \\
\hline & 39 & Vernonia conferta & - & - & - & - & 34,0 & - & - & - & 166,5 & - & - & - & - & - & 200,5 & 184,5 & 6,59 \\
\hline & 24 & Phyllantus muellerianus & - & - & 9,5 & 48,5 & - & - & & - & - & - & - & - & - & - & 58,0 & 52,5 & 1,87 \\
\hline & 33 & Ligneux indéterminé & - & - & - & - & - & - & - & - & - & - & - & - & 68,0 & - & 68,0 & 61,0 & 2,18 \\
\hline$b$ & 35 & Cogniauxia podolaena & - & - & 2,0 & - & - & - & - & 49,5 & 154,5 & - & - & 108,0 & 76,0 & - & 390,0 & 361,0 & 12,89 \\
\hline $\mathrm{c}$ & 36 & Clerodendron splendens & - & - & - & - & - & - & - & - & - & - & - & 20,0 & 32,5 & - & 52,5 & 48 & 1,71 \\
\hline \multirow{4}{*}{ d } & 1 & Megastachya mucronata & 36,0 & 157,0 & 109,0 & 190,0 & 5,0 & $\overline{7}-$ & 90,0 & 5,0 & 82,0 & 150,0 & 78,5 & - & 71,0 & 70,5 & 1044,5 & 952,0 & 34 \\
\hline & 2 & Paspalum conjugatum & 10,5 & 5,0 & $\overline{10}$ & 29,5 & - & 47,5 & 39,5 & 5,0 & - & 186,0 & 24,0 & - & - & - & 347,0 & 318 & 11,37 \\
\hline & 3 & Axonopus compressus & - & - & 10,5 & 23,0 & - & - & - & - & - & - & 19,0 & - & - & - & 52,5 & 48,5 & 1,73 \\
\hline & 0 & Panicum laxum & - & - & - & - & - & - & - & - & 50,5 & - & - & - & - & - & 50 & 47,5 & 1,69 \\
\hline \multirow[t]{2}{*}{ a } & 16 & Costus afer & - & - & 30,0 & - & 64,5 & - & 39,5 & 10,0 & 55,0 & - & - & - & - & 31,0 & 230,0 & 209,0 & 7,46 \\
\hline & 17 & Palisota hirsuta & - & 37,0 & 286,0 & 269,0 & 35,5 & 375,5 & - & 48,0 & 85,5 & 296,0 & - & 76,5 & 19,0 & 66,5 & 1594,5 & 1437,0 & 51,32 \\
\hline$b$ & 41 & Mussaenda landolphioides & 19,5 & - & - & - & - & 27,0 & 149,5 & -1 & 168,0 & - & 208,0 & 31,0 & - & 19,5 & 622,5 & 562,0 & 20,07 \\
\hline & 44 & Sabicea sp. & - & - & - & - & - & - & - & 56,0 & - & - & - & - & - & - & & & 1,80 \\
\hline C & 30 & Elaeis guineensis & 2,5 & 25,5 & - & 12,0 & - & - & - & - & - & 4,5 & 10,0 & - & - & - & 54,5 & 49,5 & 1,77 \\
\hline & 5 & Ligneux indéterminés & - & 14,0 & 一 & 55,0 & - & - & - & - & - & - & - & 22,5 & - & - & 91 & 85,0 & 3,03 \\
\hline Sous-tota & & Herbacées & 140,5 & 151,0 & 131,5 & 194,0 & 199,5 & 150,5 & 41,5 & 401,5 & 101,5 & 365,0 & 507,5 & 400,0 & 54,5 & 36,0 & 2874,5 & 2629,5 & 93,89 \\
\hline & & Ligneux & 0.1 & 0 & 0 & 0 & 50 & 0 & 0 & 0 & 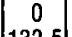 & 0 & $\begin{array}{c}0 \\
1215\end{array}$ & 0 & 0 & 0 & & & \\
\hline & & $\begin{array}{l}\text { Herbacées } \\
\text { Ligneux }\end{array}$ & $\begin{array}{c}46.5 \\
0\end{array}$ & $\mid \begin{array}{r}162,0 \\
29,0\end{array}$ & $\begin{array}{r}119,5 \\
11,5\end{array}$ & $\begin{array}{l}242,5 \\
159,0\end{array}$ & $\begin{array}{r}5,0 \\
234,0\end{array}$ & $\begin{array}{l}47.5 \\
68.5\end{array}$ & $\begin{array}{l}129,5 \\
135,5\end{array}$ & $\begin{array}{l}10,0 \\
58,5\end{array}$ & $\left|\begin{array}{l}132,5 \\
321,0\end{array}\right|$ & $\begin{array}{l}336,5 \\
237,5\end{array}$ & $\left|\begin{array}{c}121,5 \\
0\end{array}\right|$ & $\left|\begin{array}{c}0 \\
156,5\end{array}\right|$ & $\left|\begin{array}{r}71,0 \\
275,0\end{array}\right|$ & $\begin{array}{l}70,5 \\
18,5\end{array}$ & $\begin{array}{l}1494,5 \\
1704,0\end{array}$ & $\begin{array}{l}1366,5 \\
1553,5\end{array}$ & $\begin{array}{l}48,79 \\
55,46\end{array}$ \\
\hline Sous-tota & & Herbacées & 0 & 37,0 & 316,0 & 269,0 & 100,0 & 375,5 & 39,5 & 58,0 & 140,5 & 296,0 & 0 & 76,5 & 19,0 & 97,5 & 1824,5 & 1646,0 & 58,78 \\
\hline & & Ligneux & 22,0 & 39,5 & 0 & 67,0 & 0 & 27,0 & 149,5 & 56,0 & 168,0 & 4,5 & 218,0 & 53,5 & 0 & 19,5 & 824,5 & 746,5 & 26,67 \\
\hline Total & & Toutes formations & 209,0 & 381,5 & 578,5 & 931,5 & 538,5 & 668,5 & 495,5 & 584,0 & 863,5 & 1239,5 & 847,0 & 686,5 & 419,5 & 242,0 & 8722,0 & 7942,0 & 283,59 \\
\hline
\end{tabular}

TABLEAU V Participation (en p. 100) des différents types de végétation à la biomasse fourragère totale en fonction de l'áge de la plantation.

\begin{tabular}{|c|c|c|c|c|c|c|c|c|c|c|}
\hline \multicolumn{2}{|c|}{$\begin{array}{c}\text { Age des } \\
\text { palmiers (ans) }\end{array}$} & \multirow{2}{*}{\begin{tabular}{|c|}
8 \\
$\begin{array}{c}15,0 \\
0\end{array}$ \\
\end{tabular}} & \multirow{2}{*}{\begin{tabular}{|c}
9 \\
41,2 \\
0
\end{tabular}} & \multirow{2}{*}{$\begin{array}{c}10 \\
\begin{array}{c}42,0 \\
0\end{array}\end{array}$} & \multirow{2}{*}{\begin{tabular}{c|}
11 \\
28,2 \\
0
\end{tabular}} & \multirow{2}{*}{\begin{tabular}{|c|}
12 \\
29,2 \\
0
\end{tabular}} & \multirow{2}{*}{$\begin{array}{c}13 \\
20,9 \\
0\end{array}$} & \multirow{2}{*}{\begin{tabular}{|c|}
14 \\
28,5 \\
0 \\
\end{tabular}} & \multirow{2}{*}{\begin{tabular}{|c|}
15 \\
67,4 \\
0
\end{tabular}} & \multirow{2}{*}{$\begin{array}{c}\text { Ensemble } \\
\begin{array}{c}33,1 \\
0\end{array} \\
\end{array}$} \\
\hline A & $\begin{array}{l}\text { Herbacées } \\
\text { Ligneux }\end{array}$ & & & & & & & & & \\
\hline B & $\begin{array}{l}\text { Herbacées } \\
\text { Ligneux }\end{array}$ & $\begin{array}{r}29,3 \\
7,6\end{array}$ & $\begin{array}{r}6,4 \\
39,0\end{array}$ & $\begin{array}{l}22,0 \\
11,4\end{array}$ & $\begin{array}{l}14,0 \\
26,6\end{array}$ & $\begin{array}{r}4,4 \\
25,1\end{array}$ & $\begin{array}{l}26,2 \\
17,1\end{array}$ & $\begin{array}{r}28,4 \\
4,1\end{array}$ & $\begin{array}{c}22,2 \\
0\end{array}$ & $\begin{array}{l}17,2 \\
19,6\end{array}$ \\
\hline \multirow[t]{2}{*}{ C } & $\begin{array}{l}\text { Herbacées } \\
\text { Ligneux }\end{array}$ & $\begin{array}{r}40,05 \\
8,05\end{array}$ & $\begin{array}{l}8,5 \\
4,9\end{array}$ & $\begin{array}{l}14,0 \\
10,6\end{array}$ & $\begin{array}{l}12,1 \\
19,1\end{array}$ & $\begin{array}{r}39,1 \\
2,2\end{array}$ & $\begin{array}{r}28,6 \\
7,2\end{array}$ & $\begin{array}{r}35,1 \\
3,9\end{array}$ & $\begin{array}{c}0 \\
10,4\end{array}$ & $\begin{array}{r}20,7 \\
9,4\end{array}$ \\
\hline & & 100 & 100 & 100 & 100 & 100 & 100 & 100 & 100 & 100 \\
\hline & $\begin{array}{l}\text {-igneux/ } \\
+B+C \\
100 \text { du total) }\end{array}$ & 15,65 & 43,9 & 22,0 & 45,7 & 27,3 & 24,3 & 8,0 & 10,4 & 29,0 \\
\hline
\end{tabular}

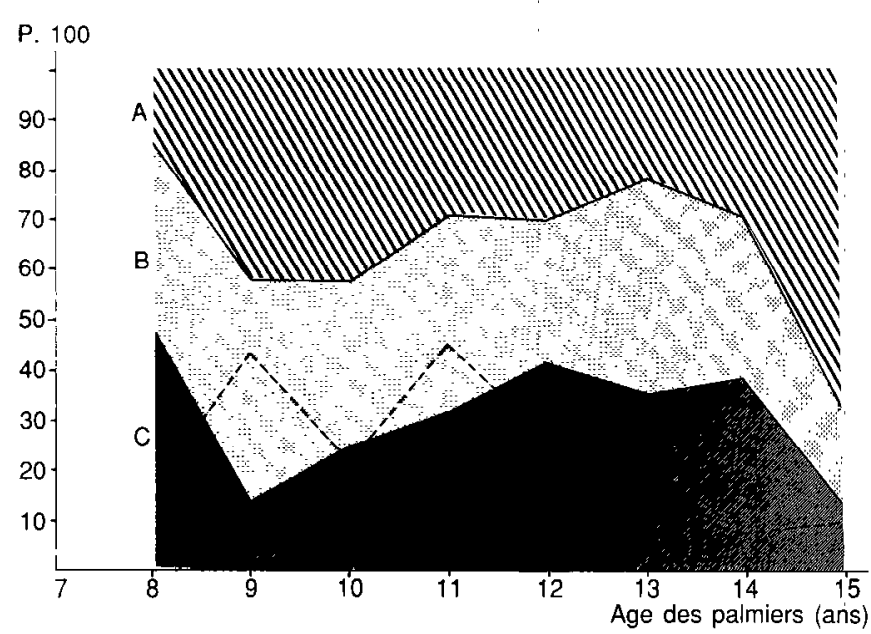

Fig. I: Part relative des trois types de végétation dans la biomasse fourragère totale en fonction de l'âge de la plantation (en pointillé, la part des ligneux dans cette même biomasse). 


\section{P. Gaullier}

cantonnées aux bordures : n'ont pas été retrouvées, mais une arbustive aussi fréquente que le parasolier (Musanga cercropioides) n'apparaît pas non plus. A défaut de pouvoir multiplier ce travail par trois ou quatre, peut-être aurait-il fallu recourir à un choix raisonné des parcelles à enquêter plutôt qu'au tirage aléatoire. II reste que, à partir de ces résultats, qui

GAULLIER (P.). Cattle's breeding in industrial oil palm in Cameroon. I. Study of the flora and biomasses measures. Revue Elev. Méd. vét. Pays trop., 1990, 43 (3) : 401-408

A cattle raising project involving the trypanosomiasis resistant N'Damia breed was started in 1979 in an industrial oil palm grove of the Littoral province, in Cameroon. A study of the pastures was undertaken in which the first part was an agrostological survey in every planting date group. This survcy gave an overall evaluation of the forage production through measures of total biomasses and useful biomasses. Influence of caring works of palm trees is examined, as well as the original features of these pastures. The study of their own dynamic and the analysis of their evolution under cattle raising effects is initiated. Key words : Cattle raising - Industrial oil palm grove Pasture - Land productivity - Biomass - Cameroon. demandent à être complétés, la prise en compte globale de l'ensemble des pâturages, réduits à leur valeur moyenne, est la seule formule possible pour une étude comparative de leur productivité et des performances des troupeaux qu'ils ont alimentés pendant quatre ans. Cette étude fait l'objet d'une sẹconde partie.

GAULLIER (P.). Ganadería vacuna en palmar industrial en Camerún. I. Estudio de la flora y medida de la biomasa. Revue Elev. Méd. vét. Pays trop., 1990, 43 (3) : 401-408

Una cría de ganado mayor de raza N'Dama, tolerante a la tripanosomosis, ha sido implantado a partir de 1979 dentro de una plantación industrial de paimerales en la província del litoral en Camerún. Se estudiaron de pastos de cual la primera fase fue una investigación agrostológica dentro de los diversos grupos de edad del palmeral ; lo que permitió hacer una evaluación global de la productividad forrajera por medida de las biomasas totales y de las biomasas útiles. Se examinan la influencia de las obras de mantenimiento de la palmeral así que las características originales de esos pastos. Se inician el estudio de su dinámica y el análisis de su evolución bajo explotación. Palabras claves: Ganado - Vacuno - Palmeral industrial - Pasto - Productividad - Biomasa - Camerún.

\section{BIBLIOGRAPHIE}

1. AUBREVILlE (A.), éd., et al. Flore du Cameroun. 1961, 31 vol. (en cours de parution).

2. AUDRU (J.). L'ćlcvage N'Dama sous palmeraies à M'Bongo et Kribi. Situation actuelle et devenir. Maisons-Alfort, IEMVT, 1985. $52 \mathrm{p}$.

3. CHOQUEI. (P.). Intérêt et utilisation des bovins trypanotolérants. Thèse doct. ENV, Maisons-Alfort, 1969, n² 22

4. COOMANS (P.), GAULLIER (P.). L'élevage bovin sous palmeraie au Cameroun. Gestion des troupeaux et pâturages. In: Productions animales tropicales au bénéfice de l'homme. Colloque intemational, Anvers, Belgique, 17-18 décembre 1982. Anvers, Institut de Médecine Tropicale Prince Léopold, 1982. P. 192-198.

5. GAULLIER (P.). L'élevage bovin sous palmeraies à la Socalpalm. Rapport de fin de projet 1979-1985. Maisons-Alfort, IEMVT, 1985. 55 p. (annexes)

6. GAULLIER (P.). Contribution de l'élevage bovin à l'entretien des plantations industrielles de palmier à huile au Cameroun. Oléagineux, 1986, 41 (6) : 255-262.

7. JACQUEMARD (P.), GUILLON (M.). La flore adventice des palmeraies adultes en Afrique équatoriale. Aperçu sur les possibilités de contrôle chimique à l'aide de nouveaux mélanges herbicides. Douala, IRHO-Shell-Cameroun, 1973.

8. KAHN (F.). La reconstitution de la forêt tropicale humide. Sud-Ouest de la Côte-d'Ivoire. Paris, ORSTOM, 1982. 88 p. (annexes et index botanique).

9. REYNOLDS (S.G.). Pâturage des bovins sous cocotiers. Revue mond. Zootech., 1980, 35: 40-45.

10. ROMBAUT (D.). Rapport au Gouvernement de la Côte-d'Ivoire sur l'élevage bovin sous palmeraies. Rome, PNUDFAO, 1973. 57 p. (AT 3229).

11. SAMUEL (C.). Cattle in oil palm. I. The effects of an integrated grazing system. Planter, 1974, 50: 201-212. 